\title{
A 12 year old girl with monoarthritis: a diagnostic dilemma
}

\author{
K A Nihal Gunatilaka ${ }^{1}$, T E Malcolm ${ }^{2}$ \\ Sri Lanka Journal of Child Health, 2004; 33: 24-6
}

(Key words: monoarthritis, child)

\section{Introduction}

When a child presents with chronic monoarthritis, the non-specific nature of the symptoms can often mislead the clinician. In such a child the following should be considered in the differential diagnosis: juvenile idiopathic arthritis (JIA), juvenile ankylosing spondylitis, juvenile psoriatic arthritis, villonodular synovitis and tuberculous arthritis.

\section{Case report}

A 11 years and 4 months old girl from Athurugiriya was referred from the paediatric surgical clinic on 02/09/2002 with a complaint of painless swelling of right knee joint (RKJ) of 1 year duration. The swelling had gradually increased in size over the past year. During the first 6 months, the parents did not seek any treatment. She was initially treated by an Ayurvedic physician with no improvement. Subsequently, she was treated thrice at the outpatients department of the Lady Ridgeway Hospital. As there was no improvement, she was referred to the surgical clinic. The white cell count, done at the surgical clinic, showed a high eosinophil count. Thus she was treated with diethyl carbamazine for 3 weeks but the swelling remained unchanged. Then she was referred to the rheumatology unit for further management.

There was no arthralgia; no limitation of movement or functional disability of the RKJ. There were no other joints involved. Constitutional symptoms were absent. There was no contact history of tuberculosis (TB). Her RKJ was grossly swollen and warm but there was no tenderness. The patellar tap was positive. The range of movement of RKJ was normal.

After initial assessment, erythrocyte sedimentation

${ }^{1}$ Consultant Rheumatologist, ${ }^{2}$ Registrar, Department of Rheumatology, Lady Ridgeway Hospital, Colombo.

(Received on 29 September 2003) rate (ESR), full blood count (FBC), blood picture (BP) and radiograph of RKJ were ordered as baseline investigations. She was given ibuprofen $200 \mathrm{mg}$ thrice daily and was requested to come back to the clinic after a week, with the investigations, for review. But she defaulted and came back to the clinic only after 3 months with no investigations, the joint swelling remaining unchanged. Thereafter baseline investigations were done. The ESR was $30 \mathrm{~mm}$ (Figure 1). The radiograph of RKJ showed only effusion.

She was then referred to an orthopaedic surgeon for synovial biopsy. Arthroscopy and synovial biopsy of the RKJ was done in January 2003. Synovial biopsy showed synovial tissue with inflammatory cell infiltration consisting of plasma cells, lymphocytes and neutrophils with focal mild synovial proliferation. This biopsy report was in favour of chronic non-specific inflammatory arthritis. Thus, a provisional diagnosis of pauciarticular JIA was made.

Following synovial biopsy, pain and limitation of movement developed in her RKJ with ligamental laxity. Since February 2003, she was treated with diclofenac sodium $25 \mathrm{mg}$ twice daily, paracetamol $500 \mathrm{mg}$ thrice daily, physiotherapy and occupational therapy. She was seen by an ophthalmologist and her eyes were found to be normal. Repeat investigations were done during this period. ESR was $44 \mathrm{~mm}$ (Figure 1). The antinuclear antibody (ANA) and rheumatoid factor (RF) were negative.

After one month of treatment arthralgia disappeared and the range of movement was back to normal but the effusion remained static. The diclofenac dose was increased to $25 \mathrm{mg}$ thrice daily. In spite of 2 months treatment RKJ effusion remained static.

During May 2003 wasting of right thigh muscle was observed and flexion of the RKJ was limited to $110^{\circ}$. Repeat ESR was $92 \mathrm{~mm}$ (Figure 1). Diclofenac was changed to indomethacin (25 mg thrice daily) with cimetidine. Methotrexate and folic acid were added at the same time. 


$\begin{array}{llllll} & \text { ESR (mm) } & \text { Hb (g\%) } & \text { WBC/DC } & \text { PLT } & \text { BP } \\ 02 / 12 / 02 & 30 & 14.2 & \begin{array}{l}8,800 \\ (\text { N39 L45 E16) }\end{array} & 464,000 & \text { NCNC } \\ & & & 10,900 & 323,000 \\ 14 / 02 / 03 & 44 & 11.8 & (\text { N62 L24 E14) } & & \\ & & & & \end{array}$

Methotrexate was introduced

$\begin{array}{llcccc}02 / 06 / 03 & 107 & 9,400 & 379,000 & \text { NCNC } \\ & & (N 58 \text { L31 E11) } & & \end{array}$

\section{After 2 months of treatment with anti TB drugs}
08/09/03
31
12.0
8,200
334,000
(N44 L38 M6 E12)

ESR - Erythrocyte Sedimentation Rate PLT - Platelet count $\quad \mathrm{Hb}$ - Haemoglobin $\quad$ BP - Blood Picture WBC/DC - White Cell Count/ Differential Count NCNC - Normochromic Normocytic

\section{Figure 1. Laboratory investigations in relation to treatment}

During June 2003 her condition deteriorated rapidly. Morning stiffness developed that lasted for 1 hour; RKJ effusion increased further in size; flexion of RKJ was restricted to $90^{\circ}$; arthralgia and limping of right leg developed. Therefore her ESR was repeated. The ESR had increased to $107 \mathrm{~mm}$ (Figure 1).

As the condition deteriorated further, methotrexate was discontinued. At this stage, we decided to review the diagnosis critically. We considered villonodular synovitis and tuberculous arthritis as the likely differential diagnoses in this girl.

Chest x-ray was normal and Mantoux test was negative. Radiograph of RKJ showed only periarticular osteoporosis. Second synovial biopsy and synovial fluid aspiration was done on 20/06/2003. Direct smear from synovial fluid showed acid-fast bacilli. DNA Amplification test (PCR) for Mycobacterium tuberculosis from synovial fluid was also positive. Synovial biopsy showed non-specific reactive changes but showed no evidence of granulomatous inflammation.

The final diagnosis arrived at was tuberculous arthritis of RKJ. She was referred to chest clinic for further management. At the chest clinic she was initially treated with rifampicin (300 mg), INAH (200 $\mathrm{mg}$ ), ethambutol (800 $\mathrm{mg}$ ) and pyrazinamide
(1000 $\mathrm{mg}$ ) for 2 months and then with rifampicin $(300 \mathrm{mg})$ and INAH $(200 \mathrm{mg})$ for 7 months.

After 2 months of treatment, RKJ effusion subsided completely. Range of movements of RKJ returned to normal. She did not have any functional disability. The ESR was $31 \mathrm{~mm}$ after 2 months of treatment (Figure 1).

\section{Discussion}

Today TB remains a major health problem in developing countries. It has re-emerged as a threat to developed countries after emergence of HIV infection ${ }^{1}$. In the past decade, there has been an increased incidence in extrapulmonary and osteoarticular manifestations worldwide ${ }^{1}$. Osteoarticular infection makes up about $5 \%$ of the cases and it predominantly affects children and young adults $^{2}$. In about $50 \%$ of these cases pulmonary involvement cannot be detected ${ }^{3}$.

Osteoarticular infection usually results from haematogenous dissemination. Spine is the most common skeletal involvement ${ }^{4}$. Joint infection may be secondary to direct invasion from an adjacent focus of tuberculous osteomyelitis. Tuberculous arthritis is typically monoarticular $(90 \%)^{3}$ and primarily involves the large weight bearing joints 
such as hip and knee ${ }^{3,4,5}$. Ankle and wrist joint involvement are uncommon.

Presentation of tuberculous arthritis is characterized by an insidious onset with a slowly progressive, painful swollen monoarthropathy ${ }^{6}$. Muscle wasting is characteristic and synovial thickening is often striking. Joint movements are restricted in all directions. Except in advanced cases, constitutional symptoms are often absent ${ }^{6}$. If the diagnosis is delayed, progression of the disease results in destruction of the joint, ankylosis and abscess or sinus formation ${ }^{2}$.

ESR is raised during the active stage. Mantoux test is virtually positive in most of the patients but a negative result does not exclude the diagnosis ${ }^{1}$. Radiograph of joint may show periarticular osteoporosis, narrow articular space, enlarged epiphysis and erosion of subarticular bone, depending on the stage of disease ${ }^{2}$. Both computerized tomography (CT) and magnetic resonance imaging (MRI) are helpful in diagnosis of tuberculous arthritis $^{7}$.

Definitive diagnosis is established with the combination of

1. Synovial fluid direct smear, culture and polymerase chain reaction (PCR) for mycobacterium tuberculosis.

2. Synovial histology and culture ${ }^{6}$.

In this patient, diagnosis was delayed because of nonspecific nature of illness. Possibility of reactivation of primary focus with the introduction of methotrexate also needs to be considered in this girl. As the diagnosis was established before the destruction of articular cartilage and bony erosion, healing will be by resolution to apparent normality.

In osteoarticular TB treatment should be at least for 9 months. First 2 months of therapy consists of INAH, rifampicin, pyrazinamide and ethambutol. After 2 months, INAH and rifampicin are given for another 7 months. Surgical intervention is done only in severe joint deformity after adequate chemotherapy ${ }^{8}$.

\section{Conclusion}

Although non-specific symptoms and the insidious nature of disease often gives diagnostic dilemma to the clinician, a high index of suspicion combined with specific investigations, timed properly, can lead to correct diagnosis at an early stage. Failure to consider tuberculosis can lead to devastating outcome otherwise preventable with today's chemotherapy. This girl's diagnosis was established ten months after she was first seen in the rheumatology clinic. Fortunately, the synovial membrane showed nonspecific reactive changes only and the girl responded well to chemotherapy and regained full function of the joint within a period of 2 months.

\section{References}

1. Elder N C. Extrapulmonary Tuberculosis: A Review. Archives of Family Medicine 1992; 1(1): $91-8$.

2. Graham A A, Louis S. Tuberculosis. In Apley's System of orthopedics and fractures; 7th ed. London; Butterworth-Heinem ann Ltd. 1993; 4750 .

3. Hurrem B, Ayse E, Hatice B, Ozlem Y, Sezer K. Multifocal Tuberculosis presenting with osteoarticular and breast involvement. Annals of Clinical Microbiology and Anti-microbials 2003; 2 (1): 6.

4. Singh S B, Saraf S K, Singh L I, Srivastava T P. Osteoarticular Tuberculosis in children; Indian Paediatrics 1992; 29 (9): 1133-7.

5. Negusse W. Bone and joint Tuberculosis in childhood in a children's hospital, Addis Ababa; Ethiopian Medical Journal 1993; 31 (1): 51-61.

6. Ellis $\mathrm{M}$ E, el-Ramahi $\mathrm{K} \mathrm{M}$, al-Dalaan A N. Tuberculosis of peripheral joints A dilemma in diagnosis; Tubercle and lung disease 1993; 74 (6): 399-404.

7. Harisinghani M G, Mcloud T C, Shepard J A, Ko J P, Shroff M M, Mueller P R. Tuberculosis from head to toe, Radiograph 2000; 20 (2): 44970 . 